\title{
Glassy magnetic ground state in layered compound $\mathrm{MnSb}_{2} \mathrm{Te}_{4}$
}

\author{
Hao $\mathrm{Li}^{1,6}$, Yaoxin $\mathrm{Li}^{3}$, Yukun $\mathrm{Lian}^{4}$, Weiwei $\mathrm{Xie}^{5}$, Ling $\mathrm{Chen}^{4}$, Jinsong Zhang ${ }^{3,7}$, Yang $\mathrm{Wu}^{2,6^{*}}$ and \\ Shoushan Fan $^{3,6}$
}

\begin{abstract}
As a sister compound of $\mathrm{MnBi}_{2} \mathrm{Te}_{4}$, the highquality $\mathrm{MnSb}_{2} \mathrm{Te}_{4}$ single crystals are grown via solid-state reaction where prolonged annealing and narrow temperature window play critical roles on account of its thermal metastability. Single-crystal X-ray diffraction (SCXRD) analysis on $\mathrm{MnSb}_{2} \mathrm{Te}_{4}$ illustrates a crystal model that is isostructural to $\mathrm{MnBi}_{2} \mathrm{Te}_{4}$, consisting of Te-Sb-Te-Mn-Te-Sb-Te septuple layers (SLs) stacking in an $\mathrm{ABC}$ sequence. However, $\mathrm{MnSb}_{2} \mathrm{Te}_{4}$ reveals a more pronounced cation intermixing in comparison with $\mathrm{MnBi}_{2} \mathrm{Te}_{4}$, comprising 28.9(7)\% Sb antisite defects on the Mn (3a) site and $19.3(6) \% \mathrm{Mn}$ antisite defects on the Sb (6c) site, which may give rise to novel magnetic properties in emerging layered $\mathrm{MnBi}_{2} \mathrm{Te}_{4}$-family materials. Unlike the antiferromagnetic (AFM) nature in $\mathrm{MnBi}_{2} \mathrm{Te}_{4}, \mathrm{MnSb}_{2} \mathrm{Te}_{4}$ exhibits a glassy magnetic ground state below $24 \mathrm{~K}$ and can be easily tuned to a ferromagnetic state under a weak applied magnetic field. Its magnetic hysteresis, anisotropy, and relaxation process are investigated in detail via static and dynamic magnetization measurements. Moreover, anomalous Hall effect as a p-type conductor is demonstrated with transport measurements. This work grants $\mathrm{MnSb}_{2} \mathrm{Te}_{4}$ a possible access to the future exploration of exotic quantum physics by removing the odd/even layer number restraint in realizing quantum transport phenomena in intrinsic $\mathrm{AFM} \mathrm{MnBi}_{2} \mathrm{Te}_{4}{ }^{-}$ family materials, as a result of the crossover between its magnetism and potential topology arising from the Sb-Te layer.
\end{abstract}

Keywords: $\mathrm{MnSb}_{2} \mathrm{Te}_{4}$, crystal growth, glassy magnetic ground state, anomalous Hall effect

\section{INTRODUCTION}

The pursuit of emerging topological materials that cultivate exotic quantum phenomena, for instance, dissipationless electronics transport and topological quantum computation, has been a research frontier in condensed matter physics and materials science [1-3]. In this regard, magnetic topological quantum materials (MTQMs) can be one of the most fascinating examples, owing to their interplay of nontrivial topology and magnetism that could give rise to numerous transport phenomena, such as the quantum anomalous Hall (QAH) effect and the axion insulator state [3-11]. One of the important examples of MTQMs is the Cr-doped $\left(\mathrm{Bi}_{x} \mathrm{Sb}_{1-x}\right)_{2} \mathrm{Te}_{3}$ thin film fabricated in a molecular epitaxy process that hosts the QAH effect [7]. However, subsequent studies on magnetic topological insulators (TIs) revealed that doping with magnetic impurity was a fairly challenging strategy in realizing homogeneous distribution in materials, restricting significant improvement in observing quantized conductance in high temperatures, and hindering the experimental progress and further application [8-13]. Therefore, intrinsic MTQMs with naturally ordered magnetic-topological interplays could serve as a cornerstone for the future discovery of numerous emerging topological properties in materials [14].

Recently, $\mathrm{MnBi}_{2} \mathrm{Te}_{4}$, a representative ternary chalcogenide with a general formula of $\mathrm{MB}_{2} \mathrm{~T}_{4}(\mathrm{M}=$ transition-metal or rareearth element, $\mathrm{B}=\mathrm{Bi}$ or $\mathrm{Sb}, \mathrm{T}=\mathrm{Te}$, Se, or $\mathrm{S}$ ), has been identified to be the first intrinsic magnetic TI by both theoretical and experimental efforts [14-32]. As a van der Waals layered compound with a tetradymite-derived structure, $\mathrm{MnBi}_{2} \mathrm{Te}_{4}$ (space group $R-3 m$ ) consists of Te-Bi-Te-Mn-Te-Bi-Te septuple layers (SLs) stacking in an $\mathrm{ABC}$ sequence. Appealingly, magnetic order and nontrivial topology inherently coexist in $\mathrm{MnBi}_{2} \mathrm{Te}_{4}$ and it belongs to MTQMs. On the one hand, each $\mathrm{Mn}^{2+}$ offers a magnetic moment of $5 \mu_{\mathrm{B}}$ according to the Hund's rule in highspin configuration. The intralayer exchange coupling between $\mathrm{Mn}-\mathrm{Mn}$ is ferromagnetic (FM) with an out-of-plane easy axis, while the interlayer coupling between neighboring SLs is antiferromagnetic (AFM), corresponding to an A-type AFM order. On the other hand, the Bi-Te layers could generate topological states by their strong spin-orbit coupling. The magnetic and topological properties of $\mathrm{MnBi}_{2} \mathrm{Te}_{4}$ together generate a gapped surface state by symmetry-breaking on the (0001) surface, confirmed experimentally on the high-quality $\mathrm{MnBi}_{2} \mathrm{Te}_{4}$ single crystal [21]. Moreover, $\mathrm{MnBi}_{2} \mathrm{Te}_{4}$ revealed layer-numberdependent topological phase evolution, where the long-sought axion insulator phase and QAH effect have been realized in mechanically exfoliated six-SL and five-SL $\mathrm{MnBi}_{2} \mathrm{Te}_{4}$ films, respectively [31,32]. The thriving research on $\mathrm{MnBi}_{2} \mathrm{Te}_{4}$ not only contributes to the emerging topological quantum states but also

\footnotetext{
${ }^{1}$ School of Materials Science and Engineering, Tsinghua University, Beijing 100084, China

${ }^{2}$ Department of Mechanical Engineering, Tsinghua University, Beijing 100084, China

${ }^{3}$ State Key Laboratory of Low Dimensional Quantum Physics and Department of Physics, Tsinghua University, Beijing 100084, China

${ }^{4}$ Beijing Key Laboratory of Energy Conversion and Storage Materials, College of Chemistry, Beijing Normal University, Beijing 100875, China

${ }^{5}$ Department of Chemistry and Chemical Biology, Rutgers University, Piscataway 08854, USA

${ }^{6}$ Tsinghua-Foxconn Nanotechnology Research Center, Tsinghua University, Beijing 100084, China

${ }^{7}$ Frontier Science Center for Quantum Information, Beijing 100084, China

* Corresponding author (email: wuyangthu@mail.tsinghua.edu.cn)
} 
motivates the synthesis of potential MTQMs. The great variety of unexplored $\mathrm{MB}_{2} \mathrm{~T}_{4}$ family is highly possible to generate tremendous novel topological quantum phases and thus arouse further interests in quantum physics.

Herein, we report the growth of $\mathrm{MnSb}_{2} \mathrm{Te}_{4}$ single crystals, a typical member of the $\mathrm{MB}_{2} \mathrm{~T}_{4}$-family materials, and establish its property in terms of thorough characterizations of the structure, composition, thermal and magnetic analysis. Interestingly, $\mathrm{MnSb}_{2} \mathrm{Te}_{4}$ single crystal exhibits glassy behaviors below the freezing temperature $T_{\mathrm{f}} \sim 24 \mathrm{~K}$, different from the predicted and experimentally revealed A-type AFM state $\mathrm{MnBi}_{2} \mathrm{Te}_{4}$, or FM interaction reported in polycrystalline $\mathrm{MnSb}_{2} \mathrm{Te}_{4}$ [33-35]. In fact, the considerable net magnetic moment is observed in $\mathrm{MnSb}_{2} \mathrm{Te}_{4}$ single crystals below its magnetic transition temperature, which makes it a promising candidate to realize intriguing quantized conductance in zero-magnetic-field, without the restraint of layer number dependence in $\mathrm{AFM} \mathrm{MnBi}_{2} \mathrm{Te}$ [14].

\section{EXPERIMENTAL SECTION}

\section{Materials}

Sb shot (99.99\%, Alfa Aesar), Mn pieces (99.95\%, Alfa Aesar), and Te lump (99.999\%, Aladdin) were used as received.

\section{Synthesis}

MnTe precursor was synthesized by directly heating a stoichiometric mixture of high-purity Mn (99.95\%, Alfa Aesar) and Te (99.999\%, Aladdin) at $1273 \mathrm{~K}$ in a vacuum-sealed silica ampoule for three days. The ampoule was quenched in the air to obtain the $\mathrm{MnTe}$ precursor (Fig. $\mathrm{S} 1 \mathrm{~b}$ ). $\mathrm{Sb}_{2} \mathrm{Te}_{3}$ precursor was synthesized by a solid-state reaction of a stoichiometric mixture of high-purity Sb (99.99\%, Alfa Aesar) and Te (99.999\%, Aladdin). The reaction mixture was vacuum-sealed in a silica ampoule, and heated to $1073 \mathrm{~K}$ and held for $24 \mathrm{~h}$. After being slowly cooled to $863 \mathrm{~K}$ at a rate of $0.1 \mathrm{~K} \mathrm{~min}^{-1}$ and held for $48 \mathrm{~h}$, the ampoule was quenched in the air to obtain the $\mathrm{Sb}_{2} \mathrm{Te}_{3}$ precursor (Fig. S1a).

\section{Characterization}

Morphology and element analyses were carried out using an FEI NOVA SEM450 scanning electron microscope (SEM) equipped with an energy dispersive X-ray (EDX) detector. X-ray photoelectron spectroscopy (XPS) measurements were conducted using an Ulvac-Phi Quantera II X-ray photoelectron spectrometer with monochromatic $\mathrm{Al} \mathrm{Ka}$ radiation $(1486.6 \mathrm{eV})$. The $\mathrm{C} 1 \mathrm{~s}$ peak at $284.8 \mathrm{eV}$ was used as the reference. Single-crystal Xray diffraction (SCXRD) data were collected at $298 \mathrm{~K}$ on a Bruker D8 Quest diffractometer using monochromatic Mo Ka radiation $(\lambda=0.71073 \AA)$. The absorption correction was done by the multiscan method. The structure was solved by SHELXT and refined by SHELXL in OLEX2 [36-38]. Powder XRD (PXRD) patterns were collected on a Rigaku D/max-2500/PC Xray diffractometer, using $\mathrm{Cu} \mathrm{Ka}$ radiation and operating at $40 \mathrm{kV}$ and $200 \mathrm{~mA}$. Raman spectra were collected on a Horiba Jobin Yvon LabRam-HR/VV Spectrometer with a 514-nm laser source and an 1800-line grating. Differential thermal analysis (DTA) and thermogravimetric (TG) analysis were carried out on a NETZSCH STA449 F3 simultaneous thermal analyzer under $\mathrm{N}_{2}$ atmosphere. The $\mathrm{MnSb}_{2} \mathrm{Te}_{4}$ sample was enclosed in an $\mathrm{Al}_{2} \mathrm{O}_{3}$ crucible, and heated from room temperature to $973 \mathrm{~K}$ at a rate of
$10 \mathrm{~K} \mathrm{~min}^{-1}$ and then cooled to $673 \mathrm{~K}$ at a rate of $10 \mathrm{~K} \mathrm{~min}^{-1}$.

\section{Magnetic measurement}

Magnetic property measurements were carried out using a magnetic property measurement system (MPMS SQUID VSM Quantum Design). Dynamic (AC) magnetization measurements were performed with an oscillating field, which can be written as $H(t)=H_{\mathrm{DC}}+H_{\mathrm{AC}} \cos (\omega t)$,

where $t$ is the time, $\omega$ is the angular frequency, $H_{\mathrm{DC}}$ is the magnitude of static (DC) field, and $H_{\mathrm{AC}}$ is the amplitude of oscillating field $[39,40]$. The magnetization response of a measured material can be expressed as

$M(t)=M_{\mathrm{DC}}+M_{\mathrm{AC}} \cos (\omega t-\phi)$,

where $\phi$ is a phase shift due to the magnetic relaxation. AC susceptibility can be defined as

$\chi=\chi^{\prime}-\mathrm{i} \chi^{\prime \prime}$,

$\chi^{\prime}=M_{\mathrm{AC}} \cos (\phi) / H_{\mathrm{DC}}$

$\chi^{\prime \prime}=M_{\mathrm{AC}} \sin (\phi) / H_{\mathrm{DC}}$,

where the real term $\left(\chi^{\prime}\right)$ keeps in-phase with the AC field, and the imaginary term $\left(\chi^{\prime \prime}\right)$ is out-of-phase with the AC field and is a measure of the energy losses due to magnetic relaxation.

For a single relaxation time $(\tau)$, AC susceptibility can be described by the Debye model as

$\chi(\omega)=\chi+\left(\chi_{0}-\chi_{\infty}\right) /(1+\mathrm{i} \omega \tau)$,

where $\chi_{0}$ and $\chi_{\infty}$ are the susceptibility at zero frequency and extremely large frequency, respectively. In actual magnetic materials, there is a distribution for $\tau$, and a generalized Debye model:

$\chi(\omega)=\chi+\left(\chi_{0}-\chi_{\infty}\right) /(1+\mathrm{i} \omega \tau)^{(1-\alpha)}$,

where $\alpha$ is the distribution parameter for $\tau$, is used to describe the $\chi^{\prime \prime}-\chi^{\prime}$ plot (Cole-Cole plot). The derived parameters $\alpha$ and $\tau$ provide specific features of a magnetic material [39-42].

\section{Transport measurement}

Transport property measurements were carried out using a cryostat (Oxford Instruments) with a base temperature of $\sim 1.6 \mathrm{~K}$ and a magnetic field up to $2 \mathrm{~T}$. The longitudinal and Hall voltages were detected simultaneously by using Stanford Research Instrument SR830 lock-in amplifiers with an AC current generated with a Keithley 6221 current source. The $\mathrm{MnSb}_{2} \mathrm{Te}_{4}$ single crystal sample with a thickness of $\sim 35 \mu \mathrm{m}$ was obtained by mechanical exfoliation from a raw $\mathrm{MnSb}_{2} \mathrm{Te}_{4}$ single crystal by Scotch tape.

\section{RESULTS AND DISCUSSION}

\section{Crystal growth}

Learning from the thermal stability and synthetic strategy of $\mathrm{MnBi}_{2} \mathrm{Te}_{4}$ crystal [29], we adopted a similar growth strategy with extended annealing for the crystal growth of $\mathrm{MnSb}_{2} \mathrm{Te}_{4}$, predicted to be isostructural to $\mathrm{MnBi}_{2} \mathrm{Te}_{4}$. Firstly, $\mathrm{Sb}_{2} \mathrm{Te}_{3}$ and $\mathrm{MnTe}$ binary used as precursors were prepared through direct reactions of high-purity $\mathrm{Sb}$ and $\mathrm{Te}$, and $\mathrm{Mn}$ and Te in stoichiometric mixtures, respectively (EXPERIMENTAL SECTION). Hereafter, high-quality $\mathrm{MnSb}_{2} \mathrm{Te}_{4}$ single crystals well grew from a 1:1 mixture of $\mathrm{Sb}_{2} \mathrm{Te}_{3}$ and MnTe. The vacuum-sealed sample was 
heated to $1173 \mathrm{~K}$ in $9 \mathrm{~h}$ and slowly cooled to $893 \mathrm{~K}$ at a rate of $1 \mathrm{~K} \mathrm{~min}^{-1}$, followed by prolonged annealing at $893 \mathrm{~K}$ for at least 14 days. Finally, the sample was quenched in the air and cracked to pick shiny crystals by cautious selection. To remove impurities, coarse parts of crystals were carefully cut off using a scalpel or cleaved off by scotch tape under an optical microscope. The growth route can successfully afford millimeter-size $\mathrm{MnSb}_{2} \mathrm{Te}_{4}$ single crystals (Figs $1 \mathrm{a}$ and $2 \mathrm{~b}$ ).

\section{Composition and oxidation state analysis}

To get rid of the influence from ambient contamination, we prepared a fresh surface of $\mathrm{MnSb}_{2} \mathrm{Te}_{4}$ crystals by cleaving them with scotch tape. The SEM image of the exfoliated $\mathrm{MnSb}_{2} \mathrm{Te}_{4}$ crystal shows a smooth surface (Fig. 1a). Distinctive edges between $\mathrm{MnSb}_{2} \mathrm{Te}_{4}$ layers of different thicknesses can be observed, confirming it is a van der Waals layered compound. Therefore, it is reasonable to obtain high-quality few-SL $\mathrm{MnSb}_{2} \mathrm{Te}_{4}$ flakes via mechanical exfoliation. EDX spectroscopy was further adopted to analyze the composition. The EDX spectrum (Fig. S2a) displays no peaks other than Sb, Mn, Te, and C (from carbon tape). In addition, the corresponding EDX elemental mapping images in Fig. 1b-d clearly show a uniform elemental distribution of Sb, Mn, and Te. Quantification based on the EDX spectrum using $\mathrm{Sb} \mathrm{L}$, Te $\mathrm{L}$, and $\mathrm{Mn} \mathrm{K}$ edges gives the composition as $\mathrm{Mn} 15.6, \mathrm{Sb} 26.9$, Te 57.4 at\%, corresponding to the chemical formula of $\mathrm{Mn}_{1.1} \mathrm{Sb}_{1.9} \mathrm{Te}_{4.0}$.

XPS measurements were carried out on the exfoliated fresh surface of $\mathrm{MnSb}_{2} \mathrm{Te}_{4}$ crystal to determine the oxidation states. The XPS survey spectrum (Fig. S2b) consolidates the constituent elements as $\mathrm{Sb}, \mathrm{Mn}$, and $\mathrm{Te}$. In the high-resolution $\mathrm{Sb} 3 \mathrm{~d}$

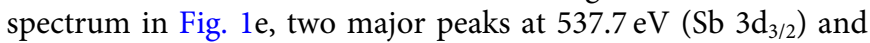
$528.3 \mathrm{eV}\left(\mathrm{Sb} 3 \mathrm{~d}_{5 / 2}\right)$ can be assigned to $\mathrm{Sb}^{3+}$ in $\mathrm{Sb}-\mathrm{Te}$ bonds $[43,44]$. Two shoulder peaks at 539.0 and $530.0 \mathrm{eV}$ corresponding to $\mathrm{Sb}-\mathrm{O}$ bonds imply the slight oxidation due to the short exposure to air $[43,44]$. Fig. If shows the deconvoluted Mn 2p spectrum that consists of two sets of four sub peaks (Mn1('), $\operatorname{Mn} 2\left(^{\prime}\right), \operatorname{Mn} 3\left({ }^{\prime}\right)$, and Mn4(')). Mn1 and Mn1' peaks located at 639.9 and $651.4 \mathrm{eV}$ could be attributed to broken Mn-Te bonds by Te deficiency. The strongest Mn2 and Mn2' peaks at 641.0 and $653.1 \mathrm{eV}$ define the major oxidation state of $\mathrm{Mn}$ as 2+. Mn3 and $\mathrm{Mn}^{\prime}$ peaks at 642.5 and $654.8 \mathrm{eV}$, and $\mathrm{Mn} 4$ and $\mathrm{Mn} 4^{\prime}$ peaks at 645.0 and $657.9 \mathrm{eV}$ are satellite peaks of $\mathrm{Mnl}$ and $\mathrm{Mn1}^{\prime}$, and Mn2 and Mn2', respectively, which result from the charge transfer between the unfilled $3 \mathrm{~d}$ shell of $\mathrm{Mn}$ and the outer shell of $\mathrm{Te}$ in the photoelectron process, similar to $\mathrm{MnBi}_{2} \mathrm{Te}_{4}$ [45-48]. For the Te $3 \mathrm{~d}$ spectrum in Fig. 1g, it contains two peaks at $572.0 \mathrm{eV}\left(\mathrm{Te} 3 \mathrm{~d}_{5 / 2}\right)$ and $582.4 \mathrm{eV}\left(\mathrm{Te} 3 \mathrm{~d}_{3 / 2}\right)$ that can be assigned to $\mathrm{Te}^{2-}$ in $\mathrm{Mn}-\mathrm{Te}$ and $\mathrm{Sb}-\mathrm{Te}(\mathrm{Mn} / \mathrm{Sb}-\mathrm{Te})$ bonds $[43,44]$. To gain insight into its oxidation behavior, the $\mathrm{MnSb}_{2} \mathrm{Te}_{4}$ crystal was exposed to air for more than a week and investigated using XPS. As shown in Fig. S3, the $\mathrm{Sb}-\mathrm{O}$ and $\mathrm{Te}-\mathrm{O}$ signals turn out to be evidently higher than $\mathrm{Sb}-\mathrm{Te}$ and $\mathrm{Mn} / \mathrm{Sb}-\mathrm{Te}$ peaks in the high-resolution $\mathrm{Sb} 3 \mathrm{~d}$ and $\mathrm{Te} 3 \mathrm{~d}$ spectra, indicating serious surface oxidation that $\mathrm{MnSb}_{2} \mathrm{Te}_{4}$ suffers in air condition. On this account, it is necessary to keep $\mathrm{MnSb}_{2} \mathrm{Te}_{4}$ samples, especially the few-layer ones, in an inert atmosphere to avoid damage by oxygen.

\section{Crystal structure and refinement}

The crystal structure of the as-grown $\mathrm{MnSb}_{2} \mathrm{Te}_{4}$ crystal was elucidated via SCXRD measurement. As an isostructural compound to $\mathrm{MnBi}_{2} \mathrm{Te}_{4}, \mathrm{MnSb}_{2} \mathrm{Te}_{4}$ also crystallizes in the centrosymmetric space group $R \overline{3} m$ (No. 166) featuring the Te-Sb$\mathrm{Te}-\mathrm{Mn}$-Te-Sb-Te SLs stacking in an ABC sequence along the $c$ axis (Fig. 2a and Fig. S4). Table 1 lists the lattice parameters of $\mathrm{MnSb}_{2} \mathrm{Te}_{4}, a=4.2284(5) \AA$ and $c=40.705(1) \AA$, slightly smaller than those of $\mathrm{MnBi}_{2} \mathrm{Te}_{4}$. This is reasonable taking the smaller ionic radius of $\mathrm{Sb}$ than that of $\mathrm{Bi}$ into account. Besides, cation antisite disorder is ubiquitous in $\mathrm{MB}_{2} \mathrm{~T}_{4}$-type compounds $[15,35,49]$. For this reason, antisite mixing of $\mathrm{Sb}$ and $\mathrm{Mn}$ was taken into account in the structural refinement by allowing $\mathrm{Sb}$ (6c) to occupy $\mathrm{Mn}$ (3a) site and vice versa. Te vacancies were only allowed in $\mathrm{Te} 2$ position that occupied the outermost layer in the Te-Sb-Te-Mn-Te-Sb-Te SLs, because those Te atoms are easier to lose considering the weak van der Waals bonding in $\mathrm{MnSb}_{2} \mathrm{Te}_{4}$. Charge neutrality was kept in the structure refinement under the restraint of eight positive charges per formula unit by considering $\mathrm{Mn}^{\mathrm{II}}\left(\mathrm{Sb}^{\mathrm{III}}\right)_{2}\left(\mathrm{Te}^{-\mathrm{II}}\right)_{4} \mathrm{~T}$ oxidation states according to XPS results.

The refinement generated a nonstoichiometric model con-
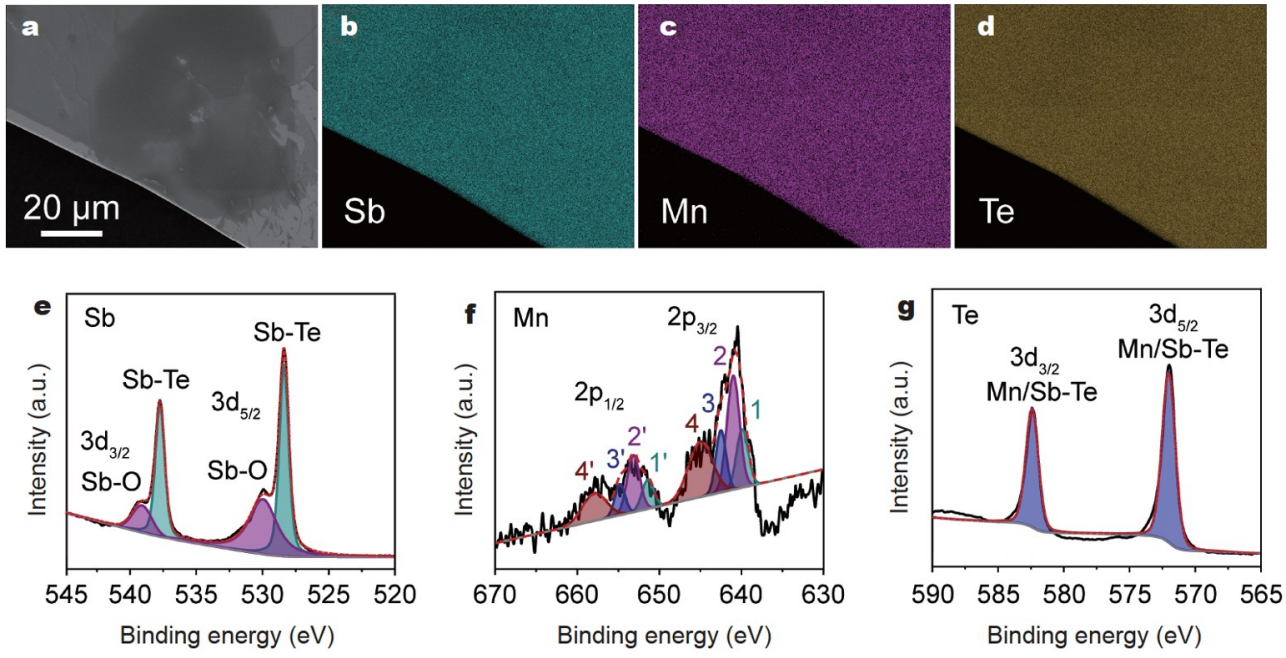

Figure 1 Morphology and elemental analyses of the exfoliated $\mathrm{MnSb}_{2} \mathrm{Te}_{4}$ single crystals. (a) SEM and (b-d) EDX elemental mapping images (Sb, Mn, and $\mathrm{Te}$ ). (e) Sb 3d, (f) Mn 2p, and (g) Te 3d high-resolution XPS spectra. 

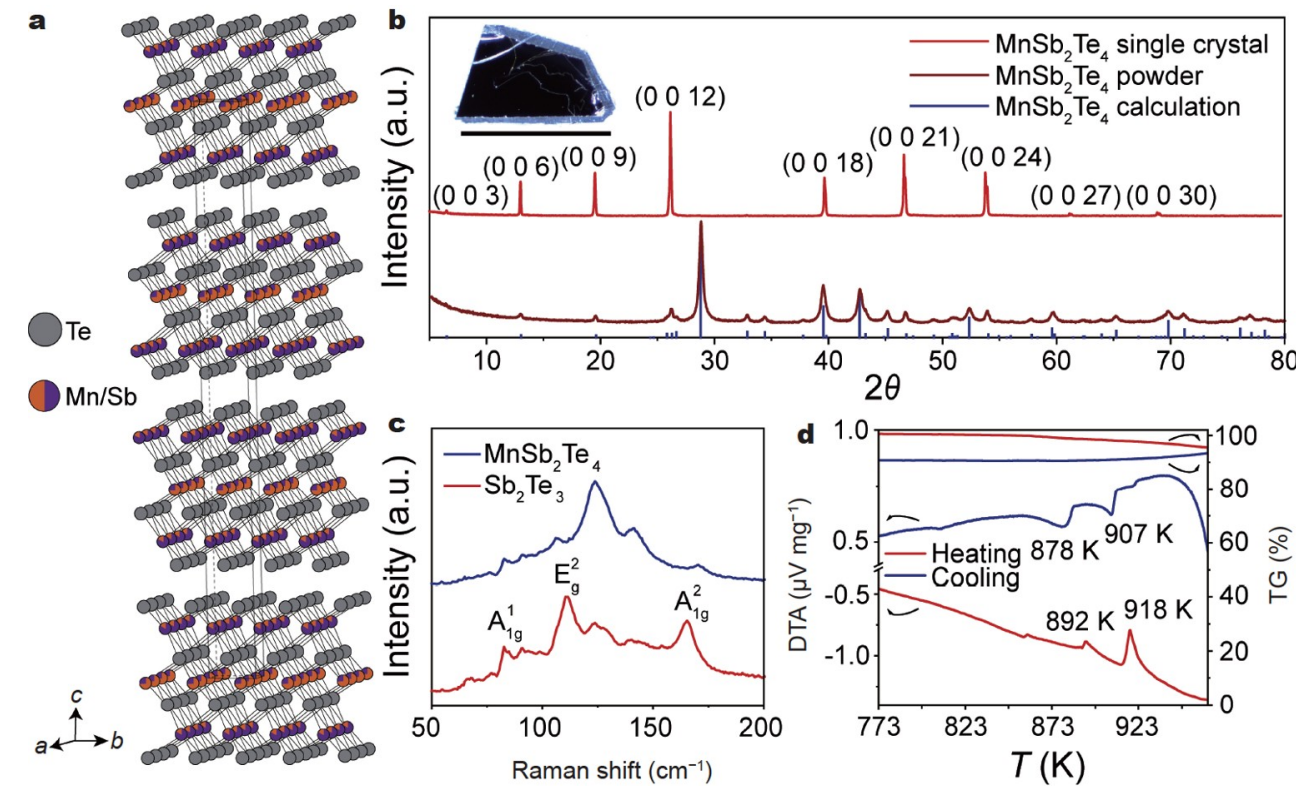

Figure 2 (a) Crystal structure of the $\mathrm{Mn}_{1.09(7)} \mathrm{Sb}_{1.90(3)} \mathrm{Te}_{3.95(1)}$ model with cation intermixing. The unit cell is marked with black lines. (b) PXRD patterns of $\mathrm{MnSb}_{2} \mathrm{Te}_{4}$ single crystal (top) and powder (middle). Inset: An optical image of the as-grown $\mathrm{MnSb}_{2} \mathrm{Te}_{4}$ single crystal, and the scale bar is $2 \mathrm{~mm}$. (c) Raman spectra of $\mathrm{MnSb}_{2} \mathrm{Te}_{4}$ and $\mathrm{Sb}_{2} \mathrm{Te}_{3}$. (d) DTA and TG analysis curves of $\mathrm{MnSb}_{2} \mathrm{Te}_{4}$.

Table 1 Crystallographic data of $\mathrm{Mn}_{1.09(7)} \mathrm{Sb}_{1.90(3)} \mathrm{Te}_{3.95(1)}$, refined with cation intermixing and Te vacancies from SCXRD

\begin{tabular}{cc}
\hline Empirical formula & $\mathrm{Mn}_{1.09(7)} \mathrm{Sb}_{1.90(3)} \mathrm{Te}_{3.95(1)}$ \\
\hline Formula units & $Z=3$ \\
Crystal system, space group & Trigonal, $R \overline{3} m$ (no. 166) \\
Lattice parameters & $a=4.2284(5) \AA$ \\
& $c=40.705(10) \AA$ \\
& $V=630.3(2) \AA^{3}$ \\
Temperature for SCXRD & $304.36 \mathrm{~K}$ \\
The range for data collection & $6.006^{\circ} \leq 2 \theta \leq 60.982^{\circ}$ \\
& $(\lambda=0.71073 \AA)$ \\
Index ranges & $-5 \leq h \leq 6,-6 \leq k \leq 6$, \\
Collected reflections & $-57 \leq l \leq 57$ \\
$R$ indexes of merging & 3406 measured, 286 unique \\
Data/restraints/parameters & $R_{\text {int }}=0.1012, R_{\sigma}=0.0364$ \\
Final $R$ indexes $[I>2 \sigma(I)]$ & $286 / 1 / 15$ \\
Final $R$ indexes (all data) & $R_{1}=0.0526, w R_{2}=0.1346$ \\
Goodness-of-fit on $F^{2}$ & $R_{1}=0.0564, w R_{2}=0.1386$ \\
Min./max. residual electron density & 1.130 \\
\hline
\end{tabular}

sisting of 28.9(7)\% Sb occupancy on the Mn (3a) site, $19.3(6) \%$ Mn occupancy on the Sb (6c) site, and 2.4(5)\% void defects on the Te2 (6c) site (Table 2). The composition that amounts to $\mathrm{Mn}_{1.09(7)} \mathrm{Sb}_{1.90(3)} \mathrm{Te}_{3.95(1)}$ is in good consistent with the SEM-EDX quantification result of $\mathrm{Mn}_{1.1} \mathrm{Sb}_{1.9} \mathrm{Te}_{4.0}$. The full structural information is shown in Tables 1 and 2, and Tables S1 and S2 in the Supplementary information (SI). For the sake of convenience, the formula $\mathrm{MnSb}_{2} \mathrm{Te}_{4}$ instead of $\mathrm{Mn}_{1.09(7)} \mathrm{Sb}_{1.90(3)} \mathrm{Te}_{3.95(1)}$ is still used throughout the article. For
Table 2 Crystallographic data of $\mathrm{Mn}_{1.09(7)} \mathrm{Sb}_{1.90(3)} \mathrm{Te}_{3.95(1)}$, refined with a cation intermixing model from an SCXRD experiment

\begin{tabular}{cccccc}
\hline Atom & $x$ & $y$ & $z$ & SOF & $U_{\text {eq }}\left(\mathrm{pm}^{2}\right)$ \\
\hline Te1 6c & $2 / 3$ & $1 / 3$ & $0.04089(3)$ & 1 & $189(4)$ \\
Te2 6c & 0 & 0 & $0.13122(3)$ & $0.975(5)$ & $188(4)$ \\
Sb1 6c & $1 / 3$ & $2 / 3$ & $0.09149(4)$ & $0.806(4)$ & $216(5)$ \\
Sb2 3a & 0 & 0 & 0 & $0.289(7)$ & $220(5)$ \\
Mn1 6c & $1 / 3$ & $2 / 3$ & $0.09149(4)$ & $0.193(6)$ & $216(5)$ \\
Mn2 3a & 0 & 0 & 0 & $0.710(3)$ & $220(8)$ \\
\hline
\end{tabular}

comparison, the cation intermixing is in a much lighter extent in $\mathrm{MnBi}_{2} \mathrm{Te}_{4}$ prepared using the similar prolonged annealing method, which just comprises $16.0(2) \% \mathrm{Bi}$ in the $\mathrm{Mn}$ (3a) site (see Tables S3-S5 in the SI). Please note that $\mathrm{MnBi}_{2} \mathrm{Te}_{4}$ crystals grown by slow cooling within a narrow range exhibit similar antisite defects on the Mn 3a site (21.5(1)\% Bi, 73.6(1)\% Mn, and $4.9(1) \%$ voids) and $5.7(1) \%$ of Mn mixing on the Bi (6c) site [15]. Therefore, $\mathrm{MnSb}_{2} \mathrm{Te}_{4}$ affords much more pronounced cation antisite disordering than $\mathrm{MnBi}_{2} \mathrm{Te}_{4}$, and this may induce new magnetism distinct to AFM coupling.

The PXRD pattern of raw $\mathrm{MnSb}_{2} \mathrm{Te}_{4}$ single crystal in Fig. 2a displays sharp and intense peaks that follow the $\left(\begin{array}{lll}0 & 0 & l\end{array}\right), l=3 n$, diffraction rule, indicating the rhombohedral symmetry and a strongly preferred orientation along the $\left[\begin{array}{lll}0 & 0 & 1\end{array}\right]$ direction. As displayed in Fig. 2b, the PXRD pattern of ground $\mathrm{MnSb}_{2} \mathrm{Te}_{4}$ powder is in good agreement with the one calculated from the structure model of $\mathrm{Mn}_{1.09(7)} \mathrm{Sb}_{1.90(3)} \mathrm{Te}_{3.95(1)}$. No impurity peaks are observed and sharp and intense peaks are displayed, confirming the high purity and crystallinity of the as-grown $\mathrm{MnSb}_{2} \mathrm{Te}_{4}$ single crystal. The Raman spectrum of $\mathrm{MnSb}_{2} \mathrm{Te}_{4}$ crystal in Fig. $2 c$ shows an appreciable blue shift and weaker $A_{1 g}$ peaks associated with $\mathrm{Sb}_{2} \mathrm{Te}_{3}$, which might indicate some impurities that cannot be detected by PXRD [50,51]. 


\section{Thermal stability}

The thermal property of $\mathrm{MnSb}_{2} \mathrm{Te}_{4}$ was revealed using DTA. As shown in Fig. 2d, the DTA curves display an intense exothermic peak at $918 \mathrm{~K}$ on the heating trace and an endothermic peak at $907 \mathrm{~K}$ on the cooling trace, corresponding to the melting and crystallization of $\mathrm{MnSb}_{2} \mathrm{Te}_{4}$ respectively. The lower intensity of the endothermic peak at $918 \mathrm{~K}$ compared with that of the exothermic peak at $907 \mathrm{~K}$ implies that the crystallization of $\mathrm{MnSb}_{2} \mathrm{Te}_{4}$ is slow and difficult. An exothermic peak at $892 \mathrm{~K}$ on the heating trace and an endothermic peak at $878 \mathrm{~K}$ on the cooling trace can be ascribed to the melting and crystallization of $\mathrm{Sb}_{2} \mathrm{Te}_{3}$ respectively, revealing that $\mathrm{MnSb}_{2} \mathrm{Te}_{4}$ might be partly decomposed to $\mathrm{Sb}_{2} \mathrm{Te}_{3}$ during the heating. The exothermic peak at $892 \mathrm{~K}$ is considerably low while the endothermic peak at $878 \mathrm{~K}$ is stronger, suggesting that the decomposition of $\mathrm{MnSb}_{2} \mathrm{Te}_{4}$ proceeds notably faster upon the melting of $\mathrm{Sb}_{2} \mathrm{Te}_{3}$ and $\mathrm{MnSb}_{2} \mathrm{Te}_{4}$. The weight loss in the TG curve in Fig. $2 \mathrm{~d}$ could arise from the volatilization of $\mathrm{Sb}$ or Te. Accordingly, the synthesis of $\mathrm{MnSb}_{2} \mathrm{Te}_{4}$ single crystals requires strict control of the annealing temperature, balancing adequate energy for the reacting $\mathrm{MnTe}$ and $\mathrm{Sb}_{2} \mathrm{Te}_{3}$ and avoiding the severe decomposition of $\mathrm{MnSb}_{2} \mathrm{Te}_{4}$.

\section{Magnetic and transport properties}

To check the magnetic characteristics of the as-grown $\mathrm{MnSb}_{2} \mathrm{Te}_{4}$ single crystal, DC magnetization measurements were performed. Fig. $3 a$ and $b$ show the temperature dependence of magnetic susceptibility $(\chi)$ for the $\mathrm{MnSb}_{2} \mathrm{Te}_{4}$ single crystal in out-of-plane $(\boldsymbol{H} / / c)$ and in-plane $(\boldsymbol{H} / / a b)$ magnetic fields, respectively. As shown in Fig. 3a, the paramagnetic (PM) regime follows CurieWeiss law:
$\chi(T)=\chi_{0}+C /\left(T-\theta_{\mathrm{CW}}\right)$,

where $\chi_{0}$ includes core diamagnetism and Pauli paramagnetism. The Curie-Weiss fitting ( $70-250 \mathrm{~K}$ region) yields the effective magnetic moment of $\mu_{\text {eff }}$ of $5.4 \mu_{\mathrm{B}}$ by taking the relationship $C=$ $N_{\mathrm{A}} \mu_{\text {eff }}^{2} / 3 k_{\mathrm{B}}$ into account, in good consistency with the value for the high-spin state of $\mathrm{Mn}^{2+}\left(5.92 \mu_{\mathrm{B}}\right)$. At a small field of $0.01 \mathrm{~T}$, a large bifurcation between zero-field-cooled (ZFC) and fieldcooled (FC) curves and lack of a cusp in the ZFC curve in both $\boldsymbol{H} / / \boldsymbol{c}$ and $\boldsymbol{H} / / a b$ are indicative of a disordered glassy magnetic ground state below a freezing temperature $T_{\mathrm{f}} \sim 24 \mathrm{~K}[41,52,53]$. At higher fields of $0.1 \mathrm{~T}$ for $\boldsymbol{H} / / \mathrm{c}$ and $2 \mathrm{~T}$ for $\boldsymbol{H} / / a b$, ZFC and FC curves coincide, indicating it enters an FM state. The larger magnetic susceptibility in the low temperature region (5-30 K) in $\mathbf{H} / / \mathrm{c}$ than that in $\mathbf{H} / / \mathbf{a b}$ suggests a strong magnetic anisotropy and the magnetization-easy axis along the $c$ direction in $\mathrm{MnSb}_{2} \mathrm{Te}_{4}$. The magnetic anisotropy is further revealed in the field dependence of magnetization in Fig. 3c-d. The $M-H$ curves in $\mathbf{H} / / \mathrm{c}$ (Fig. 3c) display obvious hysteresis loop below $20 \mathrm{~K}$ featured by a remanent magnetization of about $0.001 \mu_{\mathrm{B}} / \mathrm{Mn}$ and a coercive field of about $0.01 \mathrm{~T}$ at $5 \mathrm{~K}$, consistent with the magnetic transition below $\sim 24 \mathrm{~K}$. Nevertheless, the linear $\mathrm{M}-\mathrm{H}$ curves in $\mathbf{H} / / \mathrm{c}$ above $30 \mathrm{~K}$ indicate the PM state. The $M-H$ curves at low fields in $\mathbf{H} / / a b$ (Fig. 3d) display linear dependence with no hysteresis, suggesting that the magnetization along the $a b$ plane is merely due to the field-bent magnetic moments originally sited along the $c$ direction. Since DC magnetization data indicate the glassy spin system of $\mathrm{MnSb}_{2} \mathrm{Te}_{4}$, the magnetic relaxation was investigated to further reveal the magnetic properties of $\mathrm{MnSb}_{2} \mathrm{Te}_{4}$. Time-dependent magnetization curves in $\mathbf{H} / / \mathrm{c}$ are
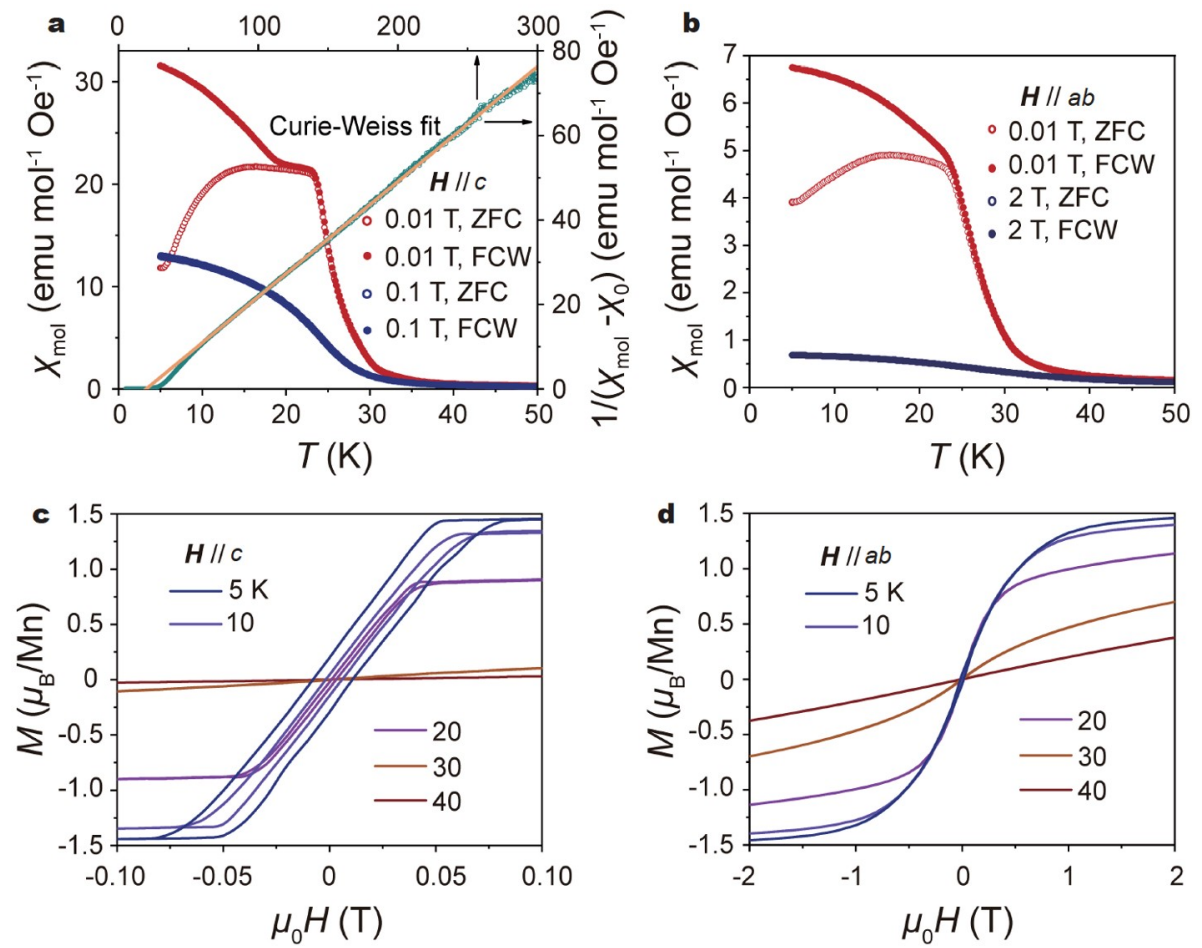

Figure 3 DC magnetization of $\mathrm{MnSb}_{2} \mathrm{Te}_{4}$ single crystal. (a, b) Temperature dependence of magnetic susceptibility measured at different fields following ZFC and FC processes, and inverse magnetic susceptibility as a function of temperature (top $x$ axis and right $y$ axis). The yellow line represents the Curie-Weiss fitting. The magnetic field is applied in out-of-plane (a) and in-plane (b) directions, respectively. (c, d) Field dependence of magnetization measured at different temperatures, and $\chi^{\prime}$ and $\chi^{\prime \prime}$ correspond to the real and imaginary part of AC magnetic susceptibility. The magnetic field is out-of-plane (c) and inplane (d), respectively. 
shown in Fig. 4a and b. In both ZFC and FC processes, magnetization continues to vary over minute-long periods. However, when the field is just increased to $0.1 \mathrm{~T}$, magnetization in ZFC process shows no relaxation, demonstrating that $\mathrm{MnSb}_{2} \mathrm{Te}_{4}$ transforms into a field-induced FM state. Fig. 4c and d display the variation of the real $\left(\chi^{\prime}\right)$ and imaginary $\left(\chi^{\prime \prime}\right)$ part of dynamic (AC) magnetic susceptibility at different frequencies as a function of temperature in $\boldsymbol{H} / / \mathrm{c}$ and $\boldsymbol{H} / / a b$, respectively. For $\boldsymbol{H} / / c$, the appearance of $\chi^{\prime \prime}$ is a clear indication of the magnetic relaxation, and the peaks of $\chi^{\prime}(T)$ and $\chi^{\prime \prime}(T)$ shift toward higher temperature as the frequency $(f)$ increases, similar to the typical behavior of a classical spin glass state [39-41,52]. Fig. S5a displays the shift of $\chi^{\prime \prime}$ peaks toward lower temperatures at a larger AC driving field of 10 Oe with respect to those at an AC driving field of $5 \mathrm{Oe}$ (Fig. 3c), illustrating that the magnetic relaxation becomes faster at a larger AC driving field and is sensitive to the amplitude of AC driving field. As shown in Fig. S5b-d, $\chi^{\prime \prime}$ is around zero and $\chi^{\prime}$ becomes frequency-independent at a DC driving field higher than $600 \mathrm{Oe}$, indicating the disappearance of magnetic relaxation. As a result, the magnetism in $\mathrm{MnSb}_{2} \mathrm{Te}_{4}$ can be turned into a field-induced FM state with a DC driving field up to $600 \mathrm{Oe}$ as affirmed by peaks in $\chi^{\prime}$, consistent with the DC magnetization results (Figs $2 \mathrm{a}$ and $3 \mathrm{a}$ ). Furthermore, as shown in Fig. S6, by fitting the frequency $(f)$ dependence of the peak shift in $\chi^{\prime}$ using $K=\Delta T_{\mathrm{f}} /\left(T_{\mathrm{f}} \Delta \log f\right), K$ is calculated to be 0.005 at $H_{\mathrm{AC}}$ $=5 \mathrm{Oe}$ and 0.007 at $H_{\mathrm{AC}}=10 \mathrm{Oe}$, in good agreement with the values $(0.0045 \leq K \leq 0.08)$ found in canonical spin glass systems [52]. For $\boldsymbol{H} / / a b, \chi^{\prime \prime}(T)$ approximates zero and peaks of $\chi^{\prime}(T)$ show no frequency-dependence, illustrating that the magnetic relaxation is weak in the in-plane direction. That is to say, the magnetization along the $a b$ plane is mainly from the field-bent magnetic moments, in good accordance with the DC magnetization results in Fig. 3 .

The distribution of magnetic relaxation time was further investigated by the Cole-Cole plot for $\chi^{\prime \prime}$ and $\chi^{\prime}$ (Fig. S7) using a generalized Debye model (EXPERIMENTAL SECTION) to obtain the $\tau$-distribution parameter $\alpha(0 \leq \alpha \leq 1)$. $\alpha$ is 0 for a single $\tau$, while $\alpha$ becomes greater for a broader distribution of $\tau$. The small derived $\alpha$ of $\mathrm{MnSb}_{2} \mathrm{Te}_{4}$ implies a rather simple magnetic relaxation process. The $\alpha$ value $(0.09$ and 0.41 at 24 and $20 \mathrm{~K}$, respectively) becomes greater with decreasing temperature, suggesting that the magnetic relaxation is largely affected by thermal energy. At a higher temperature, thermal activation could stimulate the transition between different magnetic states, generating a simpler magnetic relaxation scenario. When the temperature becomes lower, such transition needs to overcome a higher energy barrier with a more complicated magnetic relaxation process. The thermal activation of the glassy magnetic relaxation can be derived from the frequency-dependent behavior of $\chi^{\prime \prime}$. As shown in Fig. S8, the data (black dots) of $\ln (1 / \tau) v s$. $T^{-1}$ obtained at $H_{\mathrm{DC}}=0$ and $H_{\mathrm{AC}}=5,10$ Oe follow the Arrhenius law:

$\ln (1 / \tau)=-E_{\mathrm{a}} / k_{\mathrm{B}} T+\ln \left(1 / \tau_{0}\right)$,

where $\tau$ is calculated from $\tau=1 / 2 \pi f$, and $T$ is derived from peaks in $\chi^{\prime \prime}$ [39-41]. Accordingly, the effective thermal activation energy is calculated to be $579 \mathrm{meV}$ at $H_{\mathrm{AC}}=5 \mathrm{Oe}$, and $324 \mathrm{meV}$ at $H_{\mathrm{AC}}=10 \mathrm{Oe}$, suggesting that the faster and easier magnetic relaxation with a larger AC driving field.

Different from the AFM nature of $\mathrm{MnBi}_{2} \mathrm{Te}_{4}$ [15,29], $\mathrm{MnSb}_{2} \mathrm{Te}_{4}$ exhibits a glassy magnetic ground state below $T_{\mathrm{f}}$ $\sim 24 \mathrm{~K}$, which results from the much more pronounced cation
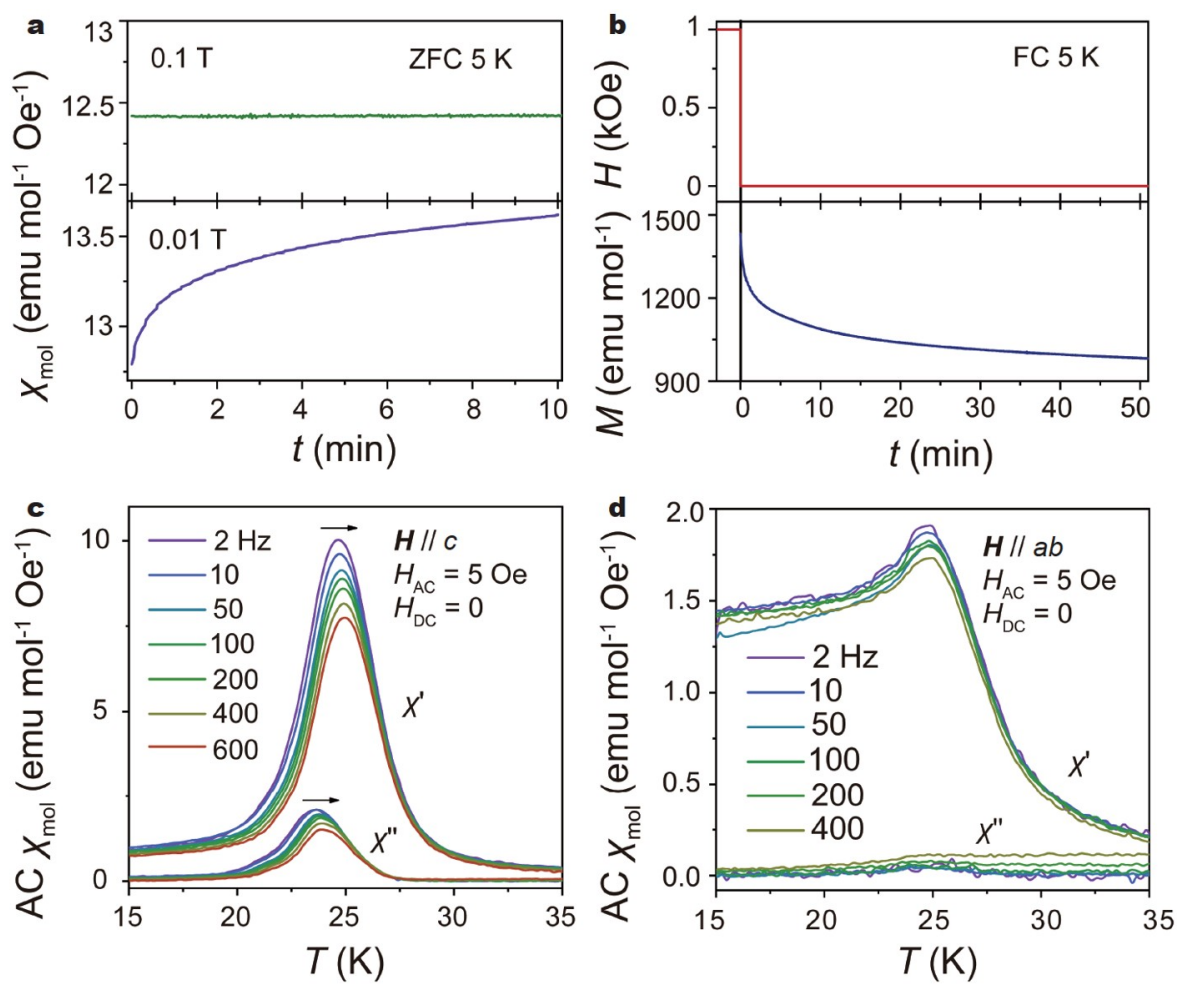

Figure 4 Magnetic relaxation of $\mathrm{MnSb}_{2} \mathrm{Te}_{4}$ single crystal. (a) Time-dependent magnetic susceptibility at $5 \mathrm{~K}$ measured at different fields following a $\mathrm{ZFC}$ process; (b) time-dependent magnetization at $5 \mathrm{~K}$ at zero field following an FC process at a field of $1 \mathrm{kOe}$; (c, d) AC magnetic susceptibility is measured at different frequencies. The magnetic field is out-of-plane (c) and in-plane (d), respectively. 

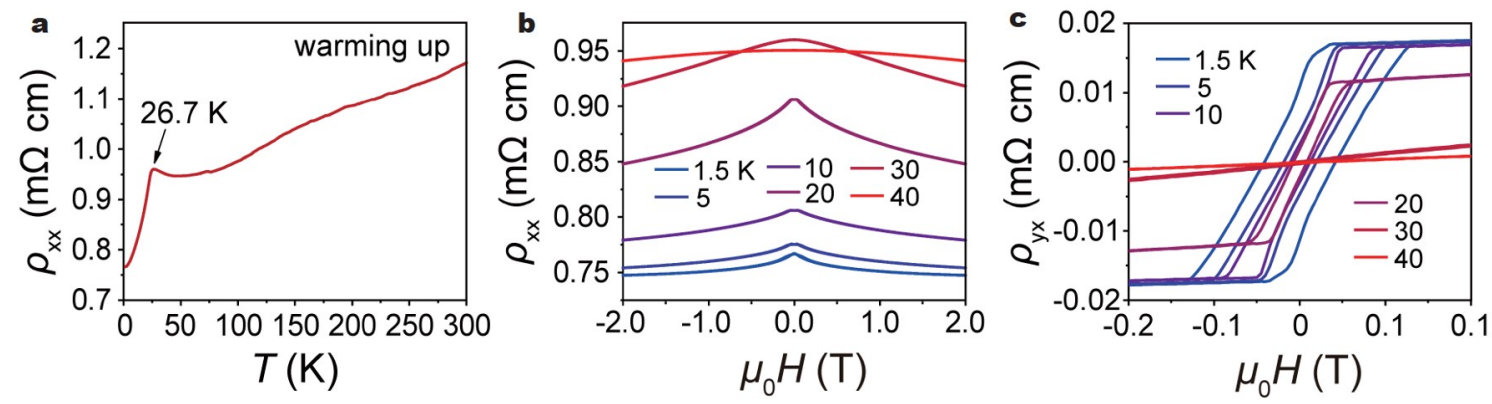

Figure 5 Transport properties of $\mathrm{MnSb}_{2} \mathrm{Te}_{4}$ single crystal. (a) Temperature dependence of longitudinal resistivity ( $\rho_{x x}$ ) measured from $1.5 \mathrm{~K}$ to room temperature; (b) MR curves in an out-of-plane magnetic field at varied temperatures from 1.5 to $40 \mathrm{~K}$; (c) magnetic field dependence of Hall resistivity ( $\rho_{y x}$ ) traces measured at the same condition as (b).

intermixing in $\mathrm{MnSb}_{2} \mathrm{Te}_{4}$ relative to that in $\mathrm{MnBi}_{2} \mathrm{Te}_{4}$. The cation intermixing as high as $28.9(7) \% \mathrm{Sb}$ on the $\mathrm{Mn}$ (3a) site and $19.3(6) \% \mathrm{Mn}$ on the $\mathrm{Sb}(6 \mathrm{c})$ site may significantly alter the $\mathrm{Mn}$ spin correlation in intra- or inter-SL layers, and lead to a frustrated ground magnetic state due to the random AFM-FM competition. Indeed, $\mathrm{MnSb}_{2} \mathrm{Te}_{4}$ can maintain considerable magnetic moment at low temperatures with no applied field as illustrated in Figs $3 \mathrm{c}$ and $4 \mathrm{~b}$. The interplay between the intrinsic magnetism and topology arising from the Sb-Te layer may lead to the realization of the zero-magnetic-field $\mathrm{QAH}$ effect in $\mathrm{MnSb}_{2} \mathrm{Te}_{4}$, breaking the odd-layer restraint in the AFM-MB $\mathrm{T}_{4}$ [14].

It is noteworthy that the magnetic ground state of $\mathrm{MnSb}_{2} \mathrm{Te}_{4}$ could be influenced by the synthesis conditions. It has been reported that the $\mathrm{MnSb}_{2} \mathrm{Te}_{4}$ polycrystalline sample synthesized via direct melting of $\mathrm{Mn}, \mathrm{Sb}$, and Te shows field-induced FM interaction [35], which is consistent with our work. In comparison, the $\mathrm{MnSb}_{2} \mathrm{Te}_{4}$ crystal grown via the Sb-Te flux method displays the AFM ground state [34], and the composition and magnetism are highly dependent on the growth condition. For instance, the $\mathrm{MnSb}_{2} \mathrm{Te}_{4}$ single crystals grown at 620 and $640^{\circ} \mathrm{C}$ exhibit AFM and FM state with an applied magnetic field of $1 \mathrm{kOe}$, respectively [54].

Furthermore, electrical transport measurements were carried out on a $\mathrm{MnSb}_{2} \mathrm{Te}_{4}$ single crystal with a thickness of $\sim 35 \mu \mathrm{m}$ to probe the transport property. Fig. 5a shows the metallic temperature dependence of longitudinal resistivity $\rho_{x x}$ in the absence of an external magnetic field from $1.5 \mathrm{~K}$ to room temperature. At high temperatures, $\rho_{x x}$ rises with increasing temperature, showing a metallic behavior. At low temperatures, an upturn of $\rho_{x x}$ takes place at $\sim 26.7 \mathrm{~K}$, which can be attributed to the change in a spin-flip scattering of charge carriers. Fig. 5b displays the magnetoresistivity (MR) curves measured in $H / / c$ at varied temperatures. The drop of MR with the increasing magnetic field is indicative of the alignment of magnetic domains. In the presence of a magnetic field, the localized magnetic moments become more aligned, reducing the scattering of charge carriers and leading to the drop of MR. Fig. $5 c$ presents the magnetic field dependence of Hall resistivity measured in $H / / c$ at varied temperatures. The positive slopes of the Hall curves demonstrate a p-type conductor with a hole density of $1.8 \times 10^{20} \mathrm{~cm}^{-3}$, and the mobility of $45 \mathrm{~cm}^{2} \mathrm{~V}^{-1} \mathrm{~s}^{-1}$ at $1.5 \mathrm{~K}$. The high carrier concentration is probably caused by the cation anti defects and $\mathrm{Te}$ vacancies. An anomalous Hall effect featured a hysteresis loop is observed, consistent with the magnetic behavior in $M-H$ curves
(Fig. 3c) and recent reports on few-layer $\mathrm{MnSb}_{2} \mathrm{Te}_{4}$ [55]. Furthermore, the demagnetization steps in the Hall curves at $1.5 \mathrm{~K}$ are a signal of relatively complicated local magnetic structures transforming from one to another, which is distinct from those of FM metals, again confirming the glassy magnetic ground state [56-58]. The demagnetization steps disappear above $5 \mathrm{~K}$, implying that the thermal energy could overcome the energy barrier of the transforming process, which is in good accordance with the magnetic measurements.

\section{CONCLUSIONS}

We developed a growth route for $\mathrm{MnSb}_{2} \mathrm{Te}_{4}$ single crystal, allowing the experimental exploration of a new member of $\mathrm{MnBi}_{2} \mathrm{Te}_{4}$-family material [29]. Similar to $\mathrm{MnBi}_{2} \mathrm{Te}_{4}$, the growth of high-quality $\mathrm{MnSb}_{2} \mathrm{Te}_{4}$ single crystals requires prolonged annealing in a narrow temperature range near $893 \mathrm{~K}$ due to its thermal metastability. The crystal structure analysis based on SCXRD data revealed the pronounced cation intermixing between $\mathrm{Mn}$ and $\mathrm{Sb}$ and led to an unexpected glassy magnetic ground state below $T_{\mathrm{f}} \sim 24 \mathrm{~K}$, accompanied with strong magnetic anisotropy with an easy axis along the $c$ direction. This magnetic behavior makes $\mathrm{MnSb}_{2} \mathrm{Te}_{4}$ quite different from $\mathrm{MnBi}_{2} \mathrm{Te}_{4}$ with a well-established AFM state. Instead, it presents a frustrated spin system owing to the FM-AFM competitions. Furthermore, $\mathrm{MnSb}_{2} \mathrm{Te}_{4}$ displays considerable magnetic moment at zero applied field, as evidenced in the hysteresis loop of $M-H$ and Hall curves in $H / / c$. The interaction of net magnetism and possible nontrivial topology in $\mathrm{MnSb}_{2} \mathrm{Te}_{4}$ enables an attractive testbed for the discovery and realization of exotic quantum phenomena without the restraint of layer number in the AFMtype $\mathrm{MnBi}_{2} \mathrm{Te}_{4}$-family materials.

Received 27 April 2021; accepted 10 June 2021;

published online 18 August 2021

1 Hasan MZ, Kane CL. Colloquium: Topological insulators. Rev Mod Phys, 2010, 82: 3045-3067

2 Qi XL, Zhang SC. Topological insulators and superconductors. Rev Mod Phys, 2011, 83: 1057-1110

3 Haldane FDM. Nobel lecture: Topological quantum matter. Rev Mod Phys, 2017, 89: 040502

$4 \mathrm{Fu} \mathrm{L}$, Kane CL. Superconducting proximity effect and Majorana fermions at the surface of a topological insulator. Phys Rev Lett, 2008, 100: 096407

5 Essin AM, Moore JE, Vanderbilt D. Magnetoelectric polarizability and axion electrodynamics in crystalline insulators. Phys Rev Lett, 2009, 
102: 146805

6 Tse WK, MacDonald AH. Giant magneto-optical Kerr effect and universal Faraday effect in thin-film topological insulators. Phys Rev Lett, 2010, 105: 057401

7 Chang CZ, Zhang J, Feng X, et al. Experimental observation of the quantum anomalous Hall effect in a magnetic topological insulator. Science, 2013, 340: 167-170

8 Chang CZ, Zhao W, Kim DY, et al. High-precision realization of robust quantum anomalous Hall state in a hard ferromagnetic topological insulator. Nat Mater, 2015, 14: 473-477

9 Katmis F, Lauter V, Nogueira FS, et al. A high-temperature ferromagnetic topological insulating phase by proximity coupling. Nature, 2016, 533: 513-516

10 Mogi M, Kawamura M, Yoshimi R, et al. A magnetic heterostructure of topological insulators as a candidate for an axion insulator. Nat Mater, 2017, 16: 516-521

11 Xiao D, Jiang J, Shin JH, et al. Realization of the axion insulator state in quantum anomalous Hall sandwich heterostructures. Phys Rev Lett, 2018, 120: 056801

12 Zhang $\mathrm{H}, \mathrm{Xu} \mathrm{Y}$, Wang J, et al. Quantum spin Hall and quantum anomalous Hall states realized in junction quantum wells. Phys Rev Lett, 2014, 112: 216803

13 Mogi M, Kawamura M, Tsukazaki A, et al. Tailoring tricolor structure of magnetic topological insulator for robust axion insulator. Sci Adv, 2017, 3: eaao1669

14 Li J, Li Y, Du S, et al. Intrinsic magnetic topological insulators in van der Waals layered $\mathrm{MnBi}_{2} \mathrm{Te}_{4}$-family materials. Sci Adv, 2019, 5: eaaw5685

15 Zeugner A, Nietschke F, Wolter AUB, et al. Chemical aspects of the candidate antiferromagnetic topological insulator $\mathrm{MnBi}_{2} \mathrm{Te}_{4}$. Chem Mater, 2019, 31: 2795-2806

16 Chen KY, Wang BS, Yan JQ, et al. Suppression of the antiferromagnetic metallic state in the pressurized $\mathrm{MnBi}_{2} \mathrm{Te}_{4}$ single crystal. Phys Rev Mater, 2019, 3: 094201

17 Chen YJ, Xu LX, Li JH, et al. Topological electronic structure and its temperature evolution in antiferromagnetic topological insulator $\mathrm{MnBi}_{2} \mathrm{Te}_{4}$. Phys Rev X, 2019, 9: 041040

18 Cui J, Shi M, Wang H, et al. Transport properties of thin flakes of the antiferromagnetic topological insulator $\mathrm{MnBi}_{2} \mathrm{Te}_{4}$. Phys Rev B, 2019, 99: 155125

19 Li J, Wang C, Zhang Z, et al. Magnetically controllable topological quantum phase transitions in the antiferromagnetic topological insulator $\mathrm{MnBi}_{2} \mathrm{Te}_{4}$. Phys Rev B, 2019, 100: 121103

20 Li Y, Jiang Z, Li J, et al. Magnetic anisotropy of the two-dimensional ferromagnetic insulator $\mathrm{MnBi}_{2} \mathrm{Te}_{4}$. Phys Rev B, 2019, 100: 134438

21 Otrokov MM, Klimovskikh II, Bentmann H, et al. Prediction and observation of an antiferromagnetic topological insulator. Nature, 2019, 576: $416-422$

22 Otrokov MM, Rusinov IP, Blanco-Rey M, et al. Unique thicknessdependent properties of the van der Waals interlayer antiferromagnet $\mathrm{MnBi}_{2} \mathrm{Te}_{4}$ Films. Phys Rev Lett, 2019, 122: 107202

23 Peng Y, Xu Y. Proximity-induced Majorana hinge modes in antiferromagnetic topological insulators. Phys Rev B, 2019, 99: 195431

24 Rani P, Saxena A, Sultana R, et al. Crystal growth and basic transport and magnetic properties of $\mathrm{MnBi}_{2} \mathrm{Te}_{4}$. J Supercond Nov Magn, 2019, 32: $3705-3709$

25 Rienks EDL, Wimmer S, Sánchez-Barriga J, et al. Large magnetic gap at the Dirac point in $\mathrm{Bi}_{2} \mathrm{Te}_{3} / \mathrm{MnBi}_{2} \mathrm{Te}_{4}$ heterostructures. Nature, 2019, 576: 423-428

26 Vidal RC, Bentmann H, Peixoto TRF, et al. Surface states and Rashbatype spin polarization in antiferromagnetic $\mathrm{MnBi}_{2} \mathrm{Te}_{4}(0001)$. Phys Rev B, 2019, 100: 121104

27 Yan JQ, Zhang Q, Heitmann T, et al. Crystal growth and magnetic structure of $\mathrm{MnBi}_{2} \mathrm{Te}_{4}$. Phys Rev Mater, 2019, 3: 064202

Zhang D, Shi M, Zhu T, et al. Topological axion states in the magnetic insulator $\mathrm{MnBi}_{2} \mathrm{Te}_{4}$ with the quantized magnetoelectric effect. Phys Rev Lett, 2019, 122: 206401

29 Li H, Liu S, Liu C, et al. Antiferromagnetic topological insulator $\mathrm{MnBi}_{2} \mathrm{Te}_{4}$ : Synthesis and magnetic properties. Phys Chem Chem Phys, 2020, 22: 556-563

30 Zhang S, Wang R, Wang X, et al. Experimental observation of the gatecontrolled reversal of the anomalous Hall effect in the intrinsic magnetic topological insulator $\mathrm{MnBi}_{2} \mathrm{Te}_{4}$ device. Nano Lett, 2020, 20: 709714

31 Liu C, Wang Y, Li H, et al. Robust axion insulator and Chern insulator phases in a two-dimensional antiferromagnetic topological insulator. Nat Mater, 2020, 19: 522-527

32 Deng Y, Yu Y, Shi MZ, et al. Quantum anomalous Hall effect in intrinsic magnetic topological insulator $\mathrm{MnBi}_{2} \mathrm{Te}_{4}$. Science, 2020, 367: 895-900

33 Chen L, Wang D, Shi C, et al. Electronic structure and magnetism of $\mathrm{MnSb}_{2} \mathrm{Te}_{4}$. J Mater Sci, 2020, 55: 14292-14300

34 Yan JQ, Okamoto S, McGuire MA, et al. Evolution of structural, magnetic, and transport properties in $\mathrm{MnBi}_{2-x} \mathrm{Sb}_{x} \mathrm{Te}_{4}$. Phys Rev B, 2019, 100: 104409

35 Murakami T, Nambu Y, Koretsune T, et al. Realization of interlayer ferromagnetic interaction in $\mathrm{MnSb}_{2} \mathrm{Te}_{4}$ toward the magnetic Weyl semimetal state. Phys Rev B, 2019, 100: 195103

36 Dolomanov OV, Bourhis LJ, Gildea RJ, et al. OLEX2: A complete structure solution, refinement and analysis program. J Appl Crystlogr, 2009, 42: 339-341

37 Sheldrick GM. SHELXT-Integrated space-group and crystal-structure determination. Acta Crystlogr A Found Adv, 2015, 71: 3-8

38 Sheldrick GM. Crystal structure refinement with SHELXL. Acta Crystlogr C Struct Chem, 2015, 71: 3-8

39 Bałanda M. Ac susceptibility studies of phase transitions and magnetic relaxation: Conventional, molecular and low-dimensional magnets. Acta Phys Pol A, 2013, 124: 964-976

40 Topping CV, Blundell SJ. A.C. susceptibility as a probe of lowfrequency magnetic dynamics. J Phys-Condens Matter, 2019, 31: 013001

41 Binder K, Young AP. Spin glasses: Experimental facts, theoretical concepts, and open questions. Rev Mod Phys, 1986, 58: 801-976

42 Wu J, Liu F, Liu C, et al. Toward 2D magnets in the $\left(\mathrm{MnBi}_{2} \mathrm{Te}_{4}\right)$ $\left(\mathrm{Bi}_{2} \mathrm{Te}_{3}\right)_{n}$ bulk crystal. Adv Mater, 2020, 32: 2001815

43 Zheng L, Cheng X, Cao D, et al. Direct growth of $\mathrm{Sb}_{2} \mathrm{Te}_{3}$ on graphene by atomic layer deposition. RSC Adv, 2015, 5: 40007-40011

44 Schaumann J, Loor M, Ünal D, et al. Improving the zT value of thermoelectrics by nanostructuring: Tuning the nanoparticle morphology of $\mathrm{Sb}_{2} \mathrm{Te}_{3}$ by using ionic liquids. Dalton Trans, 2017, 46: 656-668

45 Iwanowski RJ, Heinonen MH, Janik E. X-ray photoelectron spectra of zinc-blende MnTe. Chem Phys Lett, 2004, 387: 110-115

46 Iwanowski RJ, Heinonen MH, Janik E. Sputter cleaning and annealing of zinc-blende MnTe surface-XPS study. Appl Surf Sci, 2005, 249: 222-230

47 Kim W, Park IJ, Kim HJ, et al. Room-temperature ferromagnetic property in MnTe semiconductor thin film grown by molecular beam epitaxy. IEEE Trans Magn, 2009, 45: 2424-2427

48 Iwanowski RJ, Heinonen $\mathrm{MH}$, Witkowska B. X-ray photoelectron study of NiAs-type MnTe. J Alloys Compd, 2010, 491: 13-17

49 Kuropatwa BA, Kleinke H. Thermoelectric properties of stoichiometric compounds in the $(\mathrm{SnTe})_{x}\left(\mathrm{Bi}_{2} \mathrm{Te}_{3}\right)_{y}$ system. Z Anorg Allg Chem, 2012, 638: $2640-2647$

50 Gomis O, Vilaplana R, Manjón FJ, et al. Lattice dynamics of $\mathrm{Sb}_{2} \mathrm{Te}_{3}$ at high pressures. Phys Rev B, 2011, 84: 174305

51 Das D, Malik K, Deb AK, et al. Defect induced structural and thermoelectric properties of $\mathrm{Sb}_{2} \mathrm{Te}_{3}$ alloy. J Appl Phys, 2015, 118: 045102

52 Mydosh JA. Spin Glasses: An Experimental Introduction. London: Taylor \& Francis, 1993

53 Sun X, Song Y, Deng J, et al. New layered chromium chalcogenides 
$\mathrm{CsLiCrSe}_{2}, \mathrm{RbLiCrS}_{2}$ and $\mathrm{CsLiCrS}_{2}$ : Structures and properties. Dalton Trans, 2019, 48: 17572-17578

54 Liu Y, Wang LL, Zheng Q, et al. Site mixing for engineering magnetic topological insulators. Phys Rev X, 2021, 11: 021033

55 Shi G, Zhang M, Yan D, et al. Anomalous Hall effect in layered ferrimagnet $\mathrm{MnSb}_{2} \mathrm{Te}_{4}$. Chin Phys Lett, 2020, 37: 047301

56 Shang M, Chen Y, Tian G, et al. Re-entrant spin glass and stepped magnetization in mixed-valence $\mathrm{SrFe}_{3}\left(\mathrm{PO}_{4}\right)_{3}$. Phys B-Condensed Matter, 2013, 409: 42-46

57 Vlášková $\mathrm{K}$, Colman RH, Proschek $\mathrm{P}$, et al. Evidence for spin-glass ground state in fluorite-defect $\mathrm{Er}_{2} \mathrm{Zr}_{2} \mathrm{O}_{7}$ single crystals. Phys Rev B, 2019, 100: 214405

58 Liu C, Tao X, Kan X, et al. Spin-glass behavior in Co-based antiperovskite compound $\mathrm{SnNCo}_{3}$. Appl Phys Lett, 2020, 116: 052401

Acknowledgements The work was supported by the Basic Science Center Project of the National Natural Science Foundation of China (51788104), the Ministry of Science and Technology of China (2018YFA0307100), and the National Natural Science Foundation of China (51991340 and 21975140). This work at Rutgers was supported by the Beckman Young Investigator award.

Author contributions $\mathrm{Li} \mathrm{H}$ and $\mathrm{Wu} \mathrm{Y}$ conceived the idea and designed the experiments. Li $\mathrm{H}$ performed the synthesis, characterization and magnetic measurement. Li Y and Zhang J carried out the transport measurements. Lian $\mathrm{Y}$, Chen $\mathrm{L}$ and Xie $\mathrm{W}$ contributes to the SCXRD measurements and structure refinement. The paper was written by $\mathrm{Li} \mathrm{H}$ and $\mathrm{Wu}$ Y. Fan $\mathrm{S}$ supervised the study. All authors helped in the revision of the paper and contributed to the general discussion.

Conflict of interest The authors declare that they have no conflict of interest.

Supplementary information Supporting data are available in the online version of the paper.

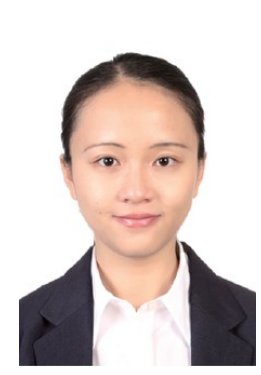

Hao Li received her BSc from the University of Science and Technology of China in 2016. She is now a doctoral candidate of materials science and engineering at Tsinghua University. Her research includes exploratory synthesis and physical properties of low-dimensional topological materials.

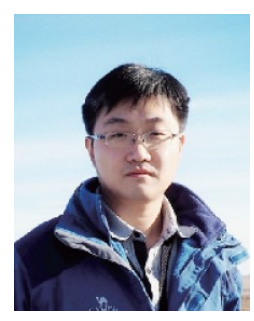

Yang $\mathbf{W u}$ received his BSc from Tsinghua University in 2002 and $\mathrm{PhD}$ from Arizona State University in 2011. He is an associate professor at the Department of Mechanical Engineering and Tsinghua-Foxconn Nanotechnology Research Center of Tsinghua University. His research interests focus on the synthesis of energy storage materials and topological quantum materials.

\section{层状化合物 $\mathrm{MnSb}_{2} \mathrm{Te}_{4}$ 中的玻璃磁基态}

李昊 ${ }^{1,6}$, 李耀金 ${ }^{3}$, 连宇坤 ${ }^{4}$, 谢韦伟 ${ }^{5}$, 陈玲 ${ }^{4}$, 张金松 3,7 , 吴扬 ${ }^{2,6^{*}}$, 范守善 3,6

摘要 作为 $\mathrm{MnBi}_{2} \mathrm{Te}_{4}$ 的姊妹化合物, 高质量的 $\mathrm{MnSb}_{2} \mathrm{Te}_{4}$ 单晶可以通过 固态反应法合成; 而由于 $\mathrm{MnSb}_{2} \mathrm{Te}_{4}$ 的热亚稳性, 长时间的退火和狭窄 的退火温区是合成的关键. 单晶X射线衍射分析结果说明 $\mathrm{MnSb}_{2} \mathrm{Te}_{4}$ 具 有与 $\mathrm{MnBi}_{2} \mathrm{Te}_{4}$ 类似的晶体结构, 由七个原子层的 $\mathrm{Te}-\mathrm{Sb}-\mathrm{Te}-\mathrm{Mn}-\mathrm{Te}-\mathrm{Sb}$ $\mathrm{Te}$ 的层状结构单元沿面外方向以 $\mathrm{ABC}$ 顺序堆叠而成. 但是, 与 $\mathrm{MnBi}_{2} \mathrm{Te}_{4}$ 相比, $\mathrm{MnSb}_{2} \mathrm{Te}_{4}$ 显示出更为严重的阳离子互占位, 表现为 $\mathrm{Mn}$ (3a)位上 的 $28.9(7) \% \mathrm{Sb}$ 反位缺陷和 $\mathrm{Sb}$ (6c)位上的 $19.3(6) \%$ 的 $\mathrm{Mn}$ 反位缺陷, 这种 原子分布的变化能够为 $\mathrm{MnBi}_{2} \mathrm{Te}_{4}$ 家族材料带来新的磁学特性. 与 $\mathrm{MnBi}_{2} \mathrm{Te}_{4}$ 的反铁磁性质不同, $\mathrm{MnSb}_{2} \mathrm{Te}_{4}$ 在低于 $24 \mathrm{~K}$ 的温度下展现出玻 璃磁基态, 并且在弱的外加磁场下很容易被诱导至铁磁态. 通过静态和 动态磁学测量, 我们详细研究了 $\mathrm{MnSb}_{2} \mathrm{Te}_{4}$ 的磁滞行为、磁各向异性和 磁弛豫过程. 此外, 通过输运测量, 我们证明了合成的 $\mathrm{MnSb}_{2} \mathrm{Te}_{4}$ 晶体是 $\mathrm{p}$ 型导体, 并且能够呈现出反常霍尔效应. $\mathrm{MnSb}_{2} \mathrm{Te}_{4}$ 的不同于 $\mathrm{MnBi}_{2} \mathrm{Te}_{4}$ 的磁学特性与 $\mathrm{Sb}-\mathrm{Te}$ 层产生的潜在拓扑特性相互作用, 有望突破反铁磁 $\mathrm{MnBi}_{2} \mathrm{Te}_{4}$ 家族材料中实现量子输运现象的奇数或者偶数的层数限制, 为新奇的量子物理学的探索带来新的可能. 